\title{
Hand Dermatitis: Review of Etiology, Diagnosis, and Treatment
}

\author{
Adam D. Perry, MD, and John P. Trafeli, MD
}

Hand dermatitis is a common condition seen in the primary care setting. Occupational exposures and frequent hand washing often lead to symptoms that are irritating and may cause discomfort. Irritant dermatitis, atopic hand dermatitis and contact hand dermatitis account for at least $\mathbf{7 0 \%}$ of all diagnoses. A unifying feature in most cases is an underlying disruption in the stratum corneum, altering its barrier function. Transepidermal water loss increases with barrier disruption and is exacerbated by additional exposure to water. Precise diagnosis and subsequent treatment present a considerable challenge, and hand dermatitis often becomes chronic. Initial treatment should be aimed at controlling inflammation and restoring the skin's natural barrier. Common management recommendations include the avoidance of irritants and potential allergens along with the use of emollients and topical corticosteroids to decrease inflammation. Simple petroleum-based emollients are very effective at restoring hydration and repairing the stratum corneum. Referral to a Dermatologist or an Allergist may be necessary for patients who require patch testing or those with refractory symptoms. (J Am Board Fam Med 2009;22: 325-30.)

Hand dermatitis represents a large proportion of occupation-associated skin disease. The prevalence among the general population has been estimated to be between $2 \%$ to $9 \%,{ }^{1}$ although one recent study cited a prevalence of $17 \%$ among a United States managed-care population, ${ }^{2}$ whereas another found that prevalence rates may be above $50 \%$ in certain occupations. ${ }^{3}$

Risk factors for hand dermatitis include the use of latex gloves, chemical exposure, and frequent handwashing. Health care professionals, machinists, housekeepers, and beauticians are examples of individuals who are at a higher risk for developing symptoms. Other predictive factors include a history of childhood eczema and female sex. Symptoms often include irritation and discomfort that, in many cases, significantly interfere with normal daily home- or work-related activities.

This article was externally peer reviewed.

Submitted 2 June 2008; revised 14 August 2008; accepted 20 August 2008.

From the United States Navy, Camp Pendleton Naval Hospital, California.

Funding: none.

Conflict of interest: The views expressed in this article are those of the authors and do not reflect the official policy or position of the Department of the Navy, Department of Defense, or the United States Government.

Corresponding author: Adam D. Perry, LT, USN, MC, MAG 39 Medical Department, Box 555750; Bldg. 22190, Camp Pendleton, CA 920555750 (E-mail: adam.perry2@ med.navy.mil).
It is estimated that $5 \%$ to $7 \%$ of patients with hand dermatitis are characterized as having chronic or severe symptoms and $2 \%$ to $4 \%$ of severe cases are refractory to traditional topical treatment. ${ }^{4}$ Management of refractory cases presents a significant challenge to the primary care provider and may require referral. Here I will review the pathogenesis, diagnosis, and treatment strategies for cases of hand dermatitis with a focus on irritant, atopic, and contact hand dermatitis.

\section{Pathogenesis}

The stratum corneum is essential in forming a barrier against the external environment and preventing water loss. This superficial layer contains epithelial cells embedded in a lipid bilayer of ceramides, fatty acids, and cholesterol with a water content between $20 \%$ and $35 \%$. Nearly all forms of hand dermatitis involve a disruption in the stratum corneum that is usually followed, but in some cases preceded, by a local inflammatory response.,

In simple terms, breeching of the stratum corneum leads to inflammatory cells being called to the scene. Inflammatory activity and transepidermal water losses lead to dryness, cracking, and inflammation. Stratum corneum lipids are mostly water soluble and water exposure from "wet work" can eliminate additional lipids. This explains the paradox regarding water making the hands drier 
along with the necessity for using emollients as a form of treatment. The drying effect of additional water may be best envisioned when thinking about cracks and hard, dry soil in a desert that are present after rain water evaporation. Water loss from the stratum corneum leads to cracks, fissures, and further impairment of its barrier function.

Disruption of the lipid bilayer in irritant hand dermatitis occurs when it is exposed to detergents, soaps, and other chemicals or irritants. Inflammation results from an irritant that is either strong enough or in contact with the skin for a long enough time to erode the barrier. Repeated or severe exposures spread to deeper layers of skin and endothelium. This, in turn, can cascade into a vicious cycle of chronic and/or severe disease. ${ }^{6}$

Underlying deficiencies within the main lipid barrier components that allow water loss are present in individuals with atopic dermatitis. These deficiencies lead to water loss, a weakened barrier, and a lower threshold for the activation of inflammation. As a result, patients have dry skin and increased vulnerability to various triggers, including both irritants and allergens. ${ }^{7}$

The mechanism in contact dermatitis is different from that noted for irritant or atopic dermatitis. Contact dermatitis involves a type IV delayed hypersensitivity reaction. Induction occurs when allergens penetrate the skin and are processed by Langerhans cells. Allergens then conjugate with carrier proteins to form antigens. Conjugated antigens migrate to lymph nodes, where sensitization occurs. Within 12 to 48 hours after re-exposure, lymphokines are released by memory T-cells and produce an inflammatory response. ${ }^{8}$

\section{Diagnostic Considerations}

Understanding each patient's disease pattern and any exacerbating factors is essential for effective diagnosis and management. Diagnostic considerations are based on history and physical examination. Initial history should consider one's occupation, history of atopic dermatitis, and handwashing activities or exposure to other irritants.

Symptoms vary according to the type of hand dermatitis. Acute symptoms in contact hand dermatitis, for example, typically consist of vesicles with superimposed weeping and crusting along with intense itching. Subacute changes often include erythema and scaling, which may lead to lichenification, fissuring, and thickening of the skin as the condition becomes chronic.

The distribution and morphology of lesions must be considered, but no distribution is classic for any one specific type of dermatitis. ${ }^{9}$ In some cases, however, an area of inflammation may correspond exactly to a region that is exposed to an allergen or irritant. As many as $20 \%$ to $35 \%$ of all dermatitis involves the hands, with many cases also involving other parts of the body. ${ }^{1}$ Therefore, it is important to ask about skin lesions in other locations to identify possible systemic dermatoses such as psoriasis.

Often, hand dermatitis is a chronic disease that is difficult to manage. Patch testing for allergens and evaluation of skin scrapings in potassium hydroxide for hyphae along with fungal and bacterial cultures may be necessary for the correct diagnosis.

\section{Differential Diagnoses}

One large study in 1990 found that the most common types of hand eczema were irritant contact dermatitis (35\%) followed by atopic hand dermatitis $(22 \%)$ and allergic contact dermatitis $(19 \%) .{ }^{10}$ The remaining diagnostic considerations are largely based on morphology and include, but are not limited to, nummular eczema, pompholyx, infections, and hyperkeratotic dermatitis. Other potential causes for hand dermatitis that are not reviewed in this article include, but are not limited to, psoriasis, id reactions, and chronic vesicular hand dermatitis.

\section{Irritant Contact Dermatitis}

This is the most common type of hand dermatitis and results from skin exposure to exogenous substances that abrade, irritate, or damage the stratum corneum. Symptoms can occur in anyone and typically include burning, itching, and tenderness at the site of exposure to an irritant. The palms, despite having a thicker stratum corneum, are frequently involved. Involvement of the fingerwebs with extension to the dorsal and ventral surfaces may also suggest this diagnosis (Figure 1). ${ }^{11}$

\section{Atopic Hand Dermatitis}

Atopic patients have a 13.5-times greater risk of developing occupational dermatoses than nonatopic patients, and represent a special category at high risk for both irritant and allergic responses. ${ }^{8}$ 


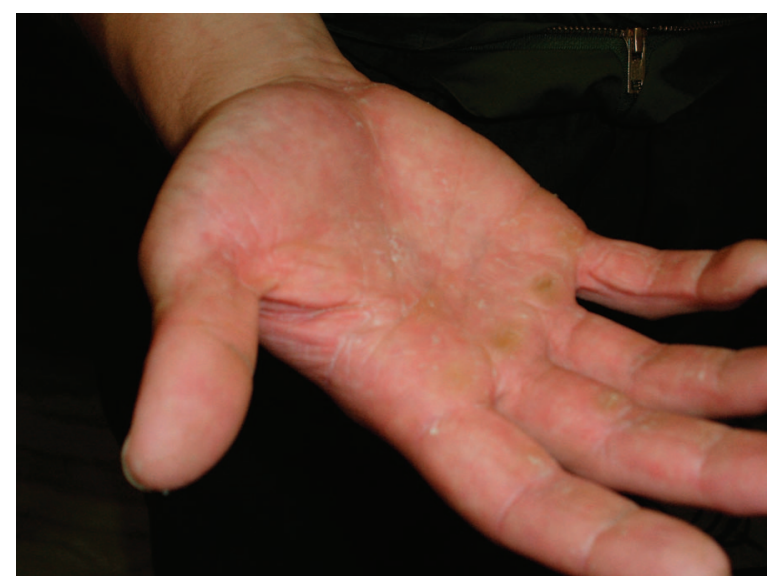

Figure 1. Irritant contact dermatitis on palms of a machinist.

Clues to the diagnosis may include hand dermatitis at a young age and frequently noted dry and pruritic skin during the patient's adult life. Symptoms tend to develop on the backs of the hands and fingers and may extend to the wrist (Figure 2).

\section{Contact Dermatitis}

Contact dermatitis, sometimes called allergic contact dermatitis, is a delayed-type hypersensitivity in which the generation of memory T-cells to an antigen will reproducibly generate an inflammatory response on rechallenge to the antigen. Substances with high sensitizing potential include nickel, rubber mix (an additive used in rubber products), fragrance mix, topical antibiotics, and potassium dichromate, along with other preservatives. These products contain low-molecular weight lipophilic

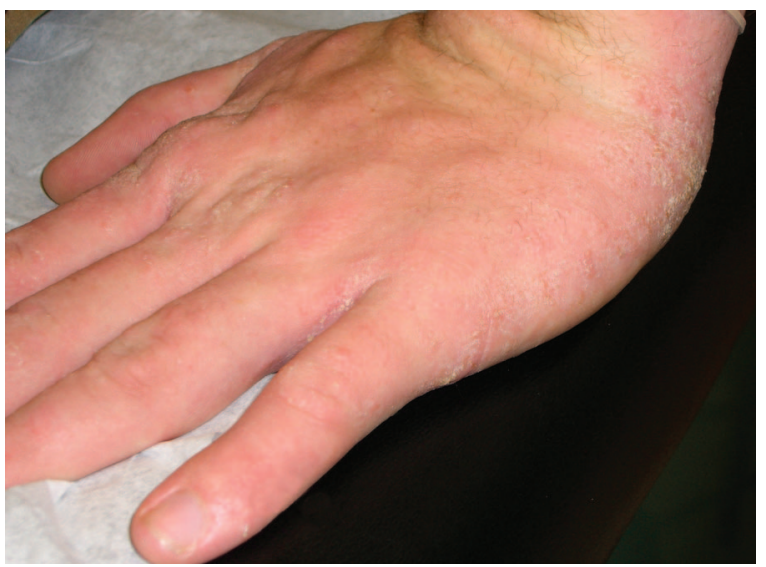

Figure 2. Atopic hand dermatitis in a patient with a history of eczema. compounds that penetrate the skin. ${ }^{12}$ Occupational risks that should alert a clinician to allergic contact dermatitis include exposure to any of these substances as well as frequent latex glove use. This may be an especially obvious consideration if there is an identifiable exposure to an object along with an associated, well-defined area of dermatitis.

The intensity of inflammation depends on the concentration of the antigen and the degree of sensitivity. Allergic contact dermatitis may favor the fingertips, nailfolds, and dorsal hands. ${ }^{13}$ Treatment must be aimed at allergen identification and avoidance as well as control of inflammation.

\section{Nummular Hand Dermatitis}

Nummular hand dermatitis is of an unknown cause and presents with circular areas of redness, scaling, and erythema that are usually seen on the backs of the hands. Symptoms may also be present on the extremities. The inflammation is often subacute or chronic and may appear psoriatic. Once present, the size of the lesions do not usually change.

\section{Pompholyx}

The term pompholyx is often used interchangeably with dishydrotic eczema; this reflects the finding that perspiration volume was found to be greater in patients with pompholyx. ${ }^{14}$ Pompholyx is the symmetric development of vesicles on the lateral aspect of the fingers preceded by pruritis. Symptoms may also be present on the palms and can also affect the feet and toes and lead to nail dystrophy. Vesicles typically last approximately 2 to 4 weeks before resolving, and then recur at varying intervals.

\section{Hyperkeratotic Dermatitis}

Hyperkeratotic dermatitis is a chronic disease that consists of symmetric dense and adherent scaling on the palmar surface. It is most common in middle aged men. The cause is not usually identifiable but may be a result of allergy or chronic irritation. The clinical course is usually chronic and stable, and treatment should resemble that used for other forms of chronic hand dermatitis. ${ }^{10}$

\section{Infections}

Gram-positive bacteria and/or fungal infections should be considered in chronic cases and in cases that do not respond to conventional treatment. In cases where an infection is suspected, culturing of vesicular or pustular lesions may be necessary, 
Table 1. Potency of Commonly Used Topical Corticosteroids $^{25}$

\begin{tabular}{ll}
\hline Group & \multicolumn{1}{c}{ Name } \\
\hline I (superpotent) & $\begin{array}{c}\text { Betamethasone dipropionate ointment, } \\
\text { cream } 0.05 \% \\
\text { Clobestasol proprionate ointment, } \\
\text { cream } 0.05 \%\end{array}$ \\
& $\begin{array}{l}\text { Fluocinonide ointment, cream/gel } \\
0.05 \%\end{array}$ \\
II & Halcinonide, cream $0.1 \%$ \\
& Betamethasone valerate ointment, \\
& $0.01 \%$ \\
III & Fluticasone propionate ointment, \\
& $0.005 \%$
\end{tabular}

along with gentle scraping of lesions for potassium hydroxide preparation to evaluate for fungal elements.

\section{Treatment}

Therapy should be individualized to the patient and must address the acute and chronic aspects of the dermatitis. Regardless of the type of hand dermatitis, restoration of the epidermal barrier is essential. $^{8}$

\section{Hydration}

Avoidance of irritants, handwashing, or other "wet work" and the use of emollients will help restore the normal skin barrier regardless of the type of hand dermatitis. Regular use of emollients and barrier creams has been shown to protect against drying and chemical irritation. ${ }^{5,15}$ Simple petroleumbased emollients are generally as effective as emollients containing skin-related lipids, although several studies suggest that topical mixtures of key stratum corneum lipids, including ceramides, may accelerate barrier repair. ${ }^{16,17}$ Adequate skin hydration is also extremely important for prevention in patients with chronic disease, even when symptoms are well controlled. Emollients are best used immediately after showering to "lock in" moisture. Skin protection programs recommending protective gloves (vinyl or cotton) and mild soaps for use when washing have been used to decrease irritant symptoms. ${ }^{18}$

\section{Identification and Avoidance}

Patch testing is important for the identification of possible contact allergens. Certain individuals may need to change jobs if symptoms are severe and if avoidance of allergens at work is difficult. ${ }^{19}$

\section{Topical Steroids}

Topical corticosteroids are often used as first-line agents to control inflammation. In general, ointments are more effective and contain fewer preservatives than creams or gels. Some authors advocate the "soak and smear" technique where mid- to high-potency steroids are applied after thorough hydration of the hands with an emollient. ${ }^{11}$ Urea can also be useful to increase topical steroid absorption, especially in chronic, lichenified cases. Acute inflammation, however, may not benefit from topical steroids because the cream or ointment may have difficulty penetrating the vesicles. Subacute inflammation requires group III to IV steroids for control (see Table 1 for a listing of topical corticosteroid potency). Chronic inflammation requires group I topical steroids without occlusion or group II to $\mathrm{V}$ topical steroids with occlusion for 1 to 3 weeks until inflammation clears. ${ }^{10}$ Occlusion is best performed using a plastic bag wrapped around the hand after applying the topical corticosteroid to an area that has been cleansed with mild soap and

Table 2. SORT: Key Recommendations for Practice

\begin{tabular}{lcc}
\hline Clinical Recommendation & Evidence Rating & References \\
\hline $\begin{array}{l}\text { Medium to high potency topical steroids are effective for initial treatment and exacerbations of } \\
\text { mild to moderate hand dermatitis }\end{array}$ & B & 16,26 \\
Petrolatum-based emollients are effective as adjuncts in the treatment of chronic hand dermatitis & B & C \\
The use of topical pimecrolimus is effective for treating chronic hand dermatitis & 21,22 \\
\hline
\end{tabular}

$\mathrm{A}=$ consistent, good-quality patient-oriented evidence; $\mathrm{B}=$ inconsistent or limited-quality patient-oriented evidence; $\mathrm{C}=$ consensus, disease-oriented evidence, usual practice, expert opinion, or case series. 
water. Continuous use of mid-potency topical steroids for longer than 3 to 4 weeks is discouraged because of possible side effects that include atrophy and telangiectasia. Tachyphylaxis also becomes more likely with continuous use and may necessitate a change in treatment.

\section{Treatment of Infections}

Antistaphylococcal antibiotics such as cephalexin or dicloxacillin are effective treatment when indicated by clinical signs and symptoms. Methicillin-resistant Staphylococcus aureus, if present, will necessitate a change in antibiotic coverage. For outpatient therapy for community-acquired Methicillin-resistant $S$. aureus skin and soft tissue infections, trimethoprim-sulfamethoxazole or doxycline should be considered. ${ }^{20}$ Bacterial culture with sensitivities should be obtained to guide therapy, help in the case of treatment failures, and document local population resistance patterns. Topical or oral antifungal medications should also be used as appropriate, depending on the results of skin scraping and subsequent potassium hydroxide preparation.

\section{Immunomodulatory Therapy}

Agents such as tacrolimus and pimecrolimus are topical substances that inhibit the release of inflammatory cytokines. Unlike topical steroids, these agents do not cause dermal atrophy or telangiectasia, and may be useful for treatment in sensitive areas and for chronic skin diseases. Pimecrolimus is thought to be more effective than tacrolimus for mild to moderate chronic hand dermatitis. ${ }^{21,22}$ The most prominent side effects are usually burning and itching at the application site, and systemic absorption and immunosuppresion are negligible. Response to these agents is slower than that for topical steroids. Both tacrolimus and pimecrolimus do however have black block warnings for rare malignancies, including skin and lymphoma, although causal relationships have not been established. Oral immunosuppresant medications such as azathioprine or methotrexate should be reserved for severe cases and managed by physicians with substantial experience with and knowledge of both the disease and the medications.

\section{Oral Steroids}

Systemic steroids may be useful in severe cases and may also be useful in short bursts for the treatment of acute vesicular symptoms or recurrent pom- pholyx. Potential side effects such as cataracts, hyperglycemia, and osteoporosis make oral steroids an undesirable choice for chronic or indiscriminatory use.

\section{Oral Antibistamines}

Oral histamine antagonists block endogenous histamine release and may alleviate pruritus. This modality may be most useful in patients with allergic hand dermatitis. $\mathrm{H} 1$ receptor antagonists are the drugs of choice.

\section{Ultraviolet Radiation Therapy}

Ultraviolet A radiation with or without psoralens has been used with at least moderate effectiveness to treat all types of resistant hand dermatitis. Ultraviolet radiation leads to local immunosuppression and decreased inflammation. Ultraviolet B radiation has been found to be equally to slightly less effective than Ultraviolet A. ${ }^{23,24}$

\section{Other Treatment Considerations}

Other available treatments include local steroid injections for resistant cases. Botulinum injections and iontophoresis have been shown to be somewhat effective for pompholyx. In cases of hyperkeratotic hand dermatitis, topical retinoids may be useful because they normalize epidermal cell maturation. $^{24}$

\section{References}

1. Warshaw EM, Ahmed RL, Belsito DV, et al. Contact dermatitis of the hands: cross-sectional analyses of North America Contact Dermatitis Group Data, 1994-2004. J Am Acad Dermatol 2007;57:301-14.

2. Fowler JF, Ghosh A, Sung J, et al. Impact of chronic hand dermatitis on quality of life, work, productivity, activity impairment, and medical costs. J Am Acad Dermatol 2006;54:448-57.

3. Lampel HP, Patel N, Boyse K, O'Brien SH, Zirwas MJ. Prevalence of hand dermatitis in inpatient nurses at a United States hospital. Dermatitis 2007;18: $140-2$.

4. Diepgen TL, Agner T, Aberer W, et al. Management of chronic hand eczema. Contact Dermatitis 2007;57:203-10.

5. Yokota M, Maiback HI. Moisturizer effect on irritant dermatitis: an overview. Contact Dermatitis 2006;55:65-72.

6. Harding CR. The stratum corneum: structure and function in health and disease. Dermatol Ther 2004; 17(Suppl 1):6-15.

7. Machedeleidt O, Kaiser HW, Sandhoff K. Defi- 
ciency of epidermal protein bound omega-hydroxyceramides in atopic dermatitis. J Invest Dermatol 2002;119:116-73.

8. Charlesworth EN. Cutaneous allergy. Cambridge: Blackwell Science;1996.

9. Cronin E. Clinical patterns of hand eczema in women. Contact Dermatitis 1985;13:153.

10. Habif TP. Clinical dermatology: a color guide to diagnosis and therapy, 4th ed. New York: Mosby; 2004.

11. Heymann WR. Hand dermatitis. J Am Acad Dermatol 2006;54:1078-80.

12. Warshaw EM. Therapeutic options for chronic hand dermatitis. Dermatol Ther 2004;17:240-50.

13. Dimsom OG. Focus on N.A.C.D.G. allergen: glutaraldehyde. Skin and Aging 2007;15:21-3.

14. Yokozeki H, Katayama I, Nishioka K, Kinoshita M, Nishiyama S. The role of metal allergy and local hyperhidrosis in the pathogenesis of pompholyx. J Dermatol 1992;19:964.

15. Ghadially R, Halkier-Sorensen L, Elias PM. Effects of petrolatum on stratum corneum structure and function. J Am Acad Dermatol 1992;26:387-96.

16. Kucherekova M, Van De Kerkhof PC, Van Der Valk PG. A randomized comparison of an emollient containing skin-related lipids with a petrolatum-based emollient as adjunct in the treatment of chronic hand dermatitis. Contact Dermatitis 2003;48:293-9.

17. Chamlin SI, Kao J, Friden IJ, et al. Ceramide-dominant barrier repair alleviate childhood atopic dermatitis: changes in barrier function provide a sensi- tive indicator of disease activity. J Am Acad Dermatol 2002;47:198-208.

18. Agner T, Held E. Skin protection programmes. Contact Dermatitis 2002;47:253-6.

19. Usmani N, Wilkinson SM. Allergic skin disease: investigation of both immediate- and delayed-type hypersensitiviy is essential. Clin Exp Allergy 2007; 37:1541-6.

20. Stevens DL, Bisno AL, Chambers HF, et al. Practice guidelines for the diagnosis and management of skin and soft-tissue infections. Clin Infect Dis 2005;41: 1373-406.

21. Belsito DV, Fowler JF Jr, Marks JG Jr, et al. Pimecrolimus cream 1\%: a potential new treatment for chronic hand dermatitis. Cutis 2004;73:31-8.

22. Thaci D, Steinmeyer K, Ebelin ME, Scott G, Kaufmann R. Occlusive treatment of chronic hand dermatitis with pimecolimus cream $1 \%$ results in low systemic exposure is well tolerated, safe and effective. Dermatology 2003;2007:37-42.

23. Meduri N, Vandegriff T, Rasmussen H, Jacobe H. Phototherapy in the management of atopic dermatitis: a systematic review. Photodermatol Photoimmunol Photomed 2007;23:106-12.

24. Veien NK, Menne T. Treatment of hand eczema. Skin Therapy Lett 2003;8:4-7.

25. Sears HW, Bailer JW, Yeadon A. Efficacy and safety of hydrocortisone buteprate $0.1 \%$ cream in patients with atopic dermatitis. Clin Ther 1997;19:710-9.

26. Veien NK, Larsen PO, Thestrup-Pedersen K, Schou G. Long-term, intermittent treatment of chronic hand eczema with mometasone furoate. Br J Dermatol $1999 ; 140: 882-6$. 